\title{
The Influence of Personal Familiarity and Context on Object Use in Semantic Dementia
}

\author{
Sasha Bozeat ${ }^{1}$, Matthew A. Lambon Ralph ${ }^{2}$, Karalyn Patterson ${ }^{1}$ and John R. Hodges ${ }^{1,3}$ \\ ${ }^{1} \mathrm{MRC}$ Cognition and Brain Sciences Unit, Cambridge, ${ }^{2}$ Department of Experimental Psychology, University of Bristol and \\ 3University Neurology Unit, Addenbrooke's Hospital, Cambridge, UK
}

\begin{abstract}
Several laboratory experiments assessing the ability of patients with semantic dementia to use familiar objects have revealed deficits corresponding directly to the patients' remaining conceptual knowledge for the same items. The same patients, however, were reported to demonstrate normal use of some objects relevant to their everyday lives. The study reported here was designed to explore this apparent discrepancy by examining the influence of personal familiarity with object exemplars, and of the contexts in which they are typically used. Two patients with severe semantic impairment were given single objects that they were still using at home on a regular basis and asked to demonstrate the use of each. Performance on these items was compared with use of perceptually similar and perceptually different exemplars of the same objects. All three sets were tested in the patients' own homes and also in the laboratory. Both patients were significantly more successful at using their own objects than the perceptually different exemplars, while an advantage for 'own' relative to similar exemplars characterized one of the two patients. Familiar home context had no impact on performance. The results suggest that repeated experience with personally familiar objects helps to maintain appropriate responses to them in the face of severely degraded conceptual knowledge.
\end{abstract}

\section{Introduction}

Patients with semantic dementia form the temporal variant of frontotemporal dementia (Snowden et al., 1989; Hodges et al., 1992; Hodges and Miller, 2001). The syndrome is associated with circumscribed temporal lobe atrophy, most severely affecting the temporal pole and the inferolateral temporal lobe, typically bilaterally but asymmetrically. The ventromedial frontal cortex bilaterally and the amygdaloid complex are also often affected (Mummery et al., 1998, 1999). The term semantic dementia was proposed because the cases have a selective and progressive deterioration of conceptual knowledge. The selective nature of the semantic impairment was first described by Warrington (1975), who reported three patients with progressive anomia and impaired word comprehension. Several more recent studies have highlighted the patients' progressive loss of knowledge about the meanings of non-verbal as well as verbal stimuli (Bozeat et al., 2000; Lambon Ralph and Howard, 2000). The selective nature of the semantic deficit in these patients has been confirmed by their good performance on assessments of current day-to-day memory, short-term verbal memory, visuospatial skills, non-verbal reasoning, phonology and syntax until very late in the course of the disease (Hodges et al., 1992).
The syndrome of semantic dementia provides an ideal testing ground for investigating the role of conceptual knowledge in various cognitive domains. These include the issue of whether the ability to use familiar objects is reliant, in whole or in part, upon intact conceptual knowledge about those items. It has been noted on the basis of clinical observation that patients with semantic dementia, despite sometimes severe semantic deficits, are relatively competent in their daily activities (Snowden et al., 1996). For instance, a number of patients with notable loss of conceptual knowledge have continued to be engaged in hobbies and/or to cook and complete various domestic chores (Hodges et al., 1992, 1998; Snowden et al., 1996; Graham et al., 1997; Lauro-Grotto et al., 1997). These cases have been reported to use a number of objects correctly, even the same objects to which they cannot provide names, descriptions or correct associative semantic judgements.

Such observations of successful completion of daily activities stand in stark contrast to the results of formal assessment of single object use in the clinic. In this situation, semantic impairment does seem to lead to impaired object use. Hodges et al. (1999) described two patients with severe loss of conceptual knowledge about objects associated with many 
failures to use the same items correctly. Hamanaka et al. (1996) also reported the co-occurrence of impoverished conceptual knowledge and impaired object use in two patients with semantic dementia. There is some indication from this report that the degree of semantic impairment is a critical factor. One of the patients initially presented with a mild semantic deficit affecting verbal comprehension and production, and at that stage had preserved object use. Over time, however, as the patient's comprehension deteriorated further, the ability to use common objects declined as well. Further evidence for this relationship comes from two additional studies. In the first (Hodges et al., 2000) the degree of impairment of nine patients in using everyday objects correlated strongly with their performance on tasks assessing conceptual knowledge for the same items. A second study of another eight patients (S. Bozeat et al., 2001, unpublished data) demonstrated and quantified the influence of three other factors on object use performance: concept familiarity, presence or absence of a typical recipient and the nature of the object's physical affordances. The clear and dramatic message from both studies is that the degree of impaired/ preserved conceptual knowledge plays a principal role in determining the success of object use.

There is also some evidence in the literature to suggest that verbal performance, object recognition and object use can all be influenced by personal familiarity, context and repeated exposure. Patients with semantic dementia continue, to some extent, to socialize and converse with others despite a profound deterioration of expressive and receptive vocabulary. Their repertoire of conversation does, however, become increasingly restricted and appears to centre around topics of current personal relevance. In a study by Snowden et al. (1994) [see also Graham et al. (1997)], patients with semantic dementia performed better on tasks involving the names of people or places that were personally relevant to them than famous names of no personal relevance. Several single case studies have established that patients of this type are able, through practice, to maintain or relearn names and pieces of information (Funnell, 1995, 2001; Graham et al., 1997), although this improved performance appears to be rigid and inflexible, and requires continued reinforcement.

With regard to performance on objects, Snowden et al. (1994) demonstrated significantly superior object recognition for a patient's own objects compared with alternative examples of the same objects. There was also an effect of context, with better recognition when objects were presented in their usual locations compared with a neutral location, which was in turn better than the performance on objects presented in an incongruous location. Object use, like the relearning of names noted above, also seems to become inflexible and restricted to the patient's own current use of the item. This is most striking when the patient's current use does not match the canonical function. For example, one patient tested by Snowden et al. $(1994,1996)$ seemed unable to report the canonical function of a jug (to pour milk, water or juice) but instead reported her own current use of that item (to hold flowers); another patient suggested that a clothes peg was used for sealing a cereal package (the patient's own use of that object in daily life) but failed to indicate its canonical function (pegging laundry).

These previous reports of preserved function for personally relevant words, information and objects can easily leave one with the impression that patients with semantic dementia are perfectly normal at home and impairments are only found once the patients cross the clinic's threshold. The inflexibility of the patients' preserved abilities is, however, an indication that performance is not normal, because normal subjects can demonstrate both generic and personally specific uses of objects and can do so with unfamiliar exemplars of known objects. S. Bozeat et al. (2001, unpublished data) found evidence that, if patients are still using objects at home, this retained ability represents a small island of preservation. As a part of that study, in addition to asking a group of control subjects to rate their familiarity with the range of test objects, we also asked the patients' spouses to confirm that their partner had previously used that item and to rate how often they used that item now. The results reinforced our clinical observations that the repertoire of tasks undertaken by these patients shrinks dramatically and includes only a small number of activities relevant to the patients' everyday needs.

Influenced by the findings of Snowden et al. (1994), we wanted to determine whether familiarity with particular exemplars of objects and the contexts in which they were typically used, had an effect on the patients' ability to use the objects. The aims of the present study were, therefore, two-fold: first, to confirm empirically that there is a difference between performance on a set of test items assessed in the clinic and the use of the patients' own examples of the same objects in their own homes; second, having established a difference, to explore two factors that might explain it: personal familiarity of the object exemplar and context. We note here that these are not the only factors that differ between formal assessment of individual objects and use of items at home for daily living. Others include the influence of computing a sequence of events to complete a task (Schwartz and Buxbaum, 1997), the importance of a goal for the task (Funnell, 2001) and the influence of other objects required to complete these tasks (e.g. recipients; S. Bozeat et al., 2001, unpublished data).

In our previous studies, which were designed to investigate the role of semantic memory in object use, we selected a cross-section of patients with varying degrees of degraded conceptual knowledge. These patients were then assessed on objects ranging from commonly used items (e.g. a knife) to those rated by a set of control subjects as less familiar (e.g. a garlic press). To maximize our chances in the current study of observing a difference between formal clinical assessment and object use at home, we employed different criteria for both patient and stimulus selection. We selected the two most semantically impaired cases from our most recent crosssectional study (S. Bozeat et al., 2001, unpublished data) so that success on formal object use assessment might be low, 
Table 1. General neuropsychology and assessment of semantic memory

\begin{tabular}{lrrr}
\hline Test (maximum score) & JH & BW & Control mean (SD) \\
\hline Digit span & & & \\
$\quad$ forward & 6 & 4 & $6.8(0.9)$ \\
$\quad$ backward & 5 & 3 & $4.7(1.2)$ \\
Letter fluency (total FAS) & 8 & 0 & $44.2(11.2)$ \\
Category fluency & & & \\
$\quad$ living & 5 & 0 & $60.3(12.6)$ \\
$\quad$ man-made & 7 & 0 & $54.8(10.3)$ \\
Naming (64) & 6 & 1 & $62.3(1.6)$ \\
Word-picture matching (64) & 18 & 8 & $63.7(0.5)$ \\
Pyramids and Palm Trees & & & \\
$\quad$ words (52) & 25 & NT & $51.1(1.1)$ \\
$\quad$ pictures (52) & 34 & 30 & $51.2(1.4)$ \\
VSP & & & \\
$\quad$ incomplete letters (20) & 17 & 19 & $19.2(0.8)$ \\
$\quad$ dot counting (10) & 10 & 10 & $9.9(0.3)$ \\
$\quad$ position discrimination (20) & 19 & 19 & $19.8(0.6)$ \\
$\quad$ cube analysis (10) & 10 & 9 & $9.7(2.5)$ \\
& & & \\
\hline
\end{tabular}

VOSP, Visual Object and Space Perception battery.

leaving room for significant benefit from, or even perhaps fully preserved use of, the patients' own objects. With regard to object selection, we started by determining which objects the patients still used at home on a regular basis and then obtained alternative versions of these items for a new formal, clinical assessment. In this way, we were able to vary personal familiarity as well as context.

\section{Case histories}

JH (born 1938), a right-handed ex-hairdresser and florist, presented in 1996 with a 3-year history of word finding difficulties. At the time of testing, JH was markedly anomic: she was able to name only six of 64 items in a picture naming test and showed severely reduced category fluency, producing just five exemplars of living things and seven man-made items (see Table 1). She was also seriously impaired on tests of comprehension, making correct responses to only 18 of 64 items in a word-picture matching task with one spoken word and 10 picture choices per trial. On the Pyramids and Palm Trees test of two-alternative forcedchoice semantic association (Howard and Patterson, 1992), JH scored $34 / 52$ on the picture condition and just 25/52 (chance) on the words. In contrast, she scored within the normal range on the digit span subtest of the Wechsler Adult Intelligence Scale (WAIS-R; Wechsler, 1981), Raven's coloured matrices (Raven, 1962) and the Visual Object and Space Perception battery (Warrington and James, 1991), indicating good working memory, non-verbal problem solving and visuospatial skills.

Despite fairly severe impairments to conceptual knowledge, at the time of this study $\mathrm{JH}$ remained competent with a number of everyday tasks around the home, although her repertoire of cooking had become limited and stereotyped. She enjoyed completing word searches and watching television, in particular quiz shows.
BW (born 1931), a right-handed ex-deputy head of a junior school, presented in 1995 with a 5-year history of word finding difficulties. At the time of testing, BW was severely anomic: she named only one of 64 items in a picture naming test and was unable to produce a single exemplar for any of the eight categories in a fluency task. Her comprehension of single concrete concepts as measured by spoken word-picture matching or picture-based semantic association was not significantly better than chance (see Table 1). In contrast, BW performed well on Raven's coloured matrices and the Visual Object and Space Perception battery, indicating preservation of non-verbal problem solving and visuospatial skills (Raven, 1962; Warrington and James, 1991).

Despite very severe semantic impairment, at the time of this study BW remained physically active and well orientated: she regularly cycled into the town on her own, and participated in swimming and aerobics classes. At home, BW spent much of her time doing word searches and jigsaw puzzles, regularly completing complex puzzles of up to 1000 pieces. She maintained the home well and was competent in the kitchen although, like $\mathrm{JH}$, her cooking repertoire had become rather limited and stereotyped.

\section{Methods \\ Object selection}

A list of kitchen objects that were used regularly by the patients was obtained from their spouses. From this list, 15 items were selected for evaluation (a different set for each patient). We used kitchen objects because they enable easy manipulation of the context in which they were assessed (see the Appendix for the lists of objects used).

\section{Personal familiarity}

Three sets of objects were included in the assessment of each patient: own, the patients' own objects, which they used regularly; perceptually similar, a set of perceptually similar exemplars of the same objects; perceptually different, a set of exemplars of the same objects selected to be as perceptually different as possible.

An example of the three conditions is given by the following: JH's own hairdryer was the standard ' $L$ ' shape, large and red, the perceptually similar one was black, slightly smaller but still the same shape, whereas the perceptually different one was a small yellow, travel hairdryer which folded up and was much 'squarer' in shape.

Given that an object's function restricts its design, the variation between the perceptually similar and perceptually different objects was typically more one of degree rather than absolute.

\section{Context}

Use of the three sets of objects was assessed in the following locations: appropriate/familiar location (in the patients' kit- 
Table 2. Examples of error type for each component of use

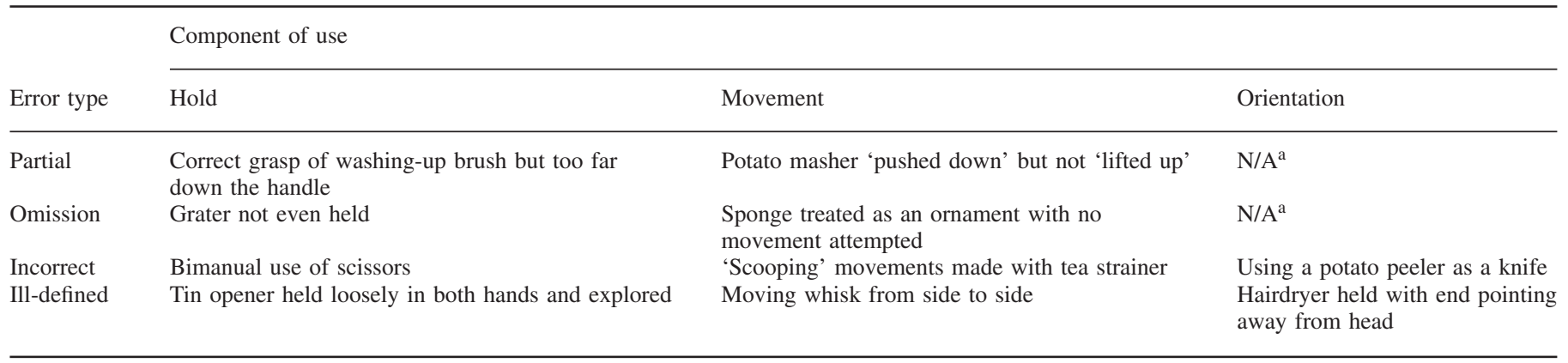

${ }^{a}$ The orientation was scored as correct or incorrect so it was not possible to observe these types of error.

chens); neutral location (in a testing room at the MRC Cognition and Brain Sciences Unit).

\section{Object use}

The patients were asked to demonstrate the use of the objects, singly. Testing took place on four different occasions, for each patient, with each session being separated by 2 weeks. The order of the items was randomized across each session and performance was videotaped for later evaluation.

\section{Scoring object use}

Following the method adopted previously for scoring the accuracy of object use (S. Bozeat et al., 2001, unpublished data), we used control subjects' demonstrations of object use to compile a list of component features that characterized object use in terms of how the object is held, manipulated and oriented. A description for the use of an object included the following components: the number of hands used to hold the object, the grasp, the position of the hand on the object, and each individual movement. So, for example, the featural description for the use of a potato masher was: held in one hand, with a 'back-standard' grasp, in the middle of the handle, with the flat end facing down, lift up, push down, movement repeated. This formed a template for scoring the patient data, producing a separate score for the hold, movement and orientation of each object. Maximum scores varied for each object, so proportional scores were calculated and averaged across the three aspects of object use for all the subsequent analyses.

\section{Error classification}

Errors were classified into one of four types: omission (no response), partially correct (only part of the appropriate full use was demonstrated), incorrect (object used as if it was another object) or ill-defined (where the object was manipulated aimlessly and the response was not recognized as suiting an alternative object). An example of each error type for each of the three components of use is given in Table 2 . It should be noted that the orientation was scored simply as correct or incorrect; it was not possible, therefore, to observe partial or omission error types for this component of use.

\section{Results}

\section{$J H$}

When demonstrating the use of objects in her own kitchen, $\mathrm{JH}$ performed well with her own objects and the perceptually similar exemplars, but was worse on the perceptually different exemplars of the same objects (see Fig. 1). When she was tested in the neutral context, a very similar pattern of results was obtained. It was interesting to note that during testing with her own objects in the laboratory, JH showed no signs of recognizing the objects as her own, although she commented on how similar each was to her own one at home.

Analysis of variance confirmed a significant main effect of object condition $\left(F_{(2,14)}=5.6, P<0.01\right)$ but no effect of context $(F<1)$ or an interaction $\left(F_{(2,14)}=1.1, P=0.33\right)$. Post-hoc tests indicated significantly better use of both her own objects and the perceptually similar exemplars than the perceptually different objects $\left(t_{(29)}=5.44, P<0.01\right.$; $\left.t_{(29)}=4.43, P<0.01\right)$. There was no significant difference between the use of JH's own objects and the perceptually similar exemplars $\left(t_{(29)}=0.98, P>0.05\right)$.

\section{$B W$}

It is clear from Fig. 2 that BW was best when using her own objects, worst when using the perceptually different exemplars, with performance somewhere in between for the perceptually similar objects. This pattern was the same in both contexts. Unlike JH, BW recognized all her own objects as being her own, even in the laboratory.

Analysis of variance confirmed a significant main effect of object condition $\left(F_{(2,14)}=8.7, P<0.001\right)$ but no effect of context $\left(F_{(1,14)}=1.1, P>0.05\right)$ or an interaction $(F<1)$. Post-hoc tests indicated that BW scored significantly higher when demonstrating the use of her own objects compared with both the perceptually similar $\left(t_{(29)}=2.03\right.$, $P=0.05)$ and the perceptually different exemplars $\left(t_{(29)}=3.36, P<0.01\right)$. She also performed significantly 


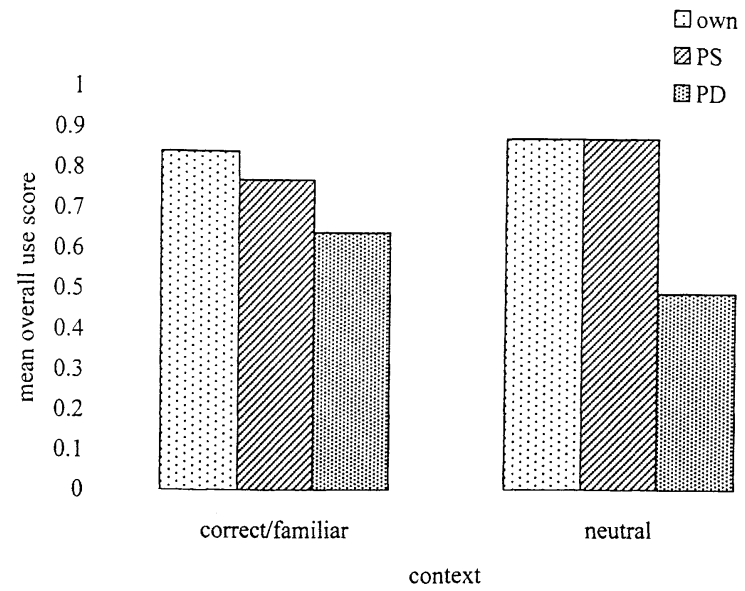

Fig. 1. Performance of $\mathrm{JH}$ on object use ( $\mathrm{PS}=$ perceptually similar; $\mathrm{PD}=$ perceptually different).

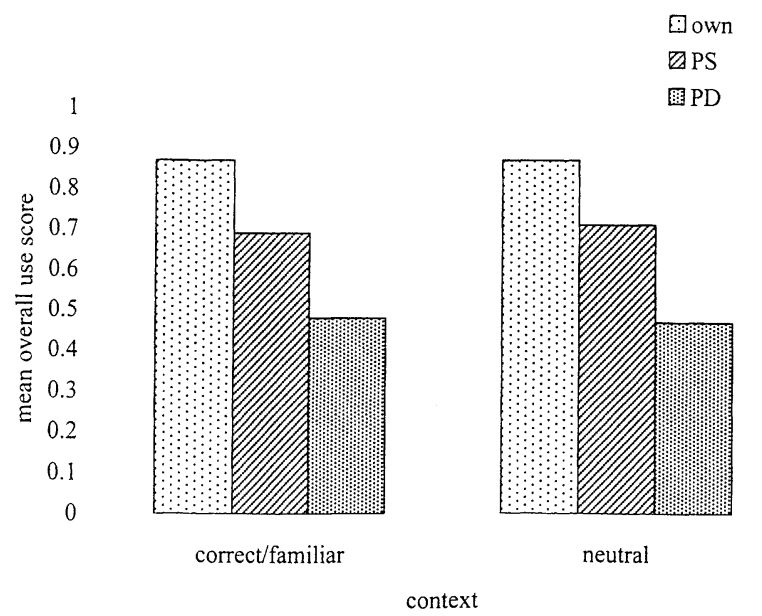

Fig. 2. Performance of $\mathrm{BW}$ on object use (PS = perceptually similar; $\mathrm{PD}=$ perceptually different).

better on the perceptually similar objects compared with the perceptually different set $\left(t_{(29)}=4.92, P<0.01\right)$.

\section{Perceptual similarity}

In this study, objects were simply categorized as perceptually similar to or perceptually different from the patients' own objects. Perceptual similarity, however, is not an all or none phenomenon, but rather a continuum. It is possible that the objects obtained for $\mathrm{JH}$ were more perceptually similar to her own objects than those used to assess BW, and that this might account for the discrepancy between the results for the two patients. In order to test this hypothesis, a featurebased analysis was used to compare the structural similarity of the perceptually similar objects and the patients' own objects. For each object a list of structural features was compiled and, as expected, there was a large overlap between the patients' own objects and the perceptually similar objects. The features were then compared on the following parameters: size, shape and colour. From this scoring scheme, a proportional score was obtained that indicated how similar each perceptually similar object was to the patient's own example. A $t$-test revealed no significant difference between the scores of perceptual similarity for the two patients $\left(t_{(28)}=1.24\right.$, $P>0.05)$.

\section{Error analysis}

Tables 3 and 4 summarize the distribution of error types produced by each patient, for each component of use-hold, movement and orientation-on the three sets of objects. The distributions were summed across the two conditions because no significant differences were found between performance in the patients' homes and the laboratory. Most of the errors made by the patients on the hold component were 'partial', with equal but small numbers of 'incorrect' and 'omission' errors; no 'ill-defined' errors were made. In contrast, the movement component was dominated by 'omission' errors, and 'partial' errors were also common, with fewer 'illdefined' and 'incorrect' errors. The orientation component elicited equal numbers of 'ill-defined' and 'incorrect' errors. No other error types were possible as this component was scored simply as correct or incorrect. There was no difference in the distribution of error types across the three different conditions (own, perceptually similar and perceptually different).

\section{Discussion}

The first aim of the present study was to confirm empirically that, at least for objects relating to daily living, there would be a significant advantage for patients with semantic dementia in using their own objects in their own homes relative to test objects assessed in the clinic. Second, having established this predicted variation in performance, we aimed to explore two factors that might explain it: personal exemplar familiarity and context. Both patients were strongly influenced by personal familiarity, while the context in which the objects were presented was not found to influence success in either patient. JH showed significantly better use of her own objects than the perceptually different items, and this enhancement also generalized to the perceptually similar exemplars. In contrast, BW's use of her own objects was superior to both the perceptually similar and the perceptually different exemplars, with a further advantage for perceptually similar objects relative to perceptually different examples.

There are several possible explanations for the disparity in results for the perceptually similar objects. The first option, already introduced and assessed in the results section, relates to the notion of graded perceptual similarity of the objects. Although it is not possible to rule out any contribution of this factor, a feature-based analysis of the objects indicated no significant difference in the level of perceptual similarity of the objects used to test the two patients. A second possibility is that normal conceptual knowledge invests us with the ability to generalize knowledge appropriately for 
Table 3. Distribution of error types observed in JH's object use (shown as percentages)

\begin{tabular}{|c|c|c|c|c|c|c|c|c|c|c|}
\hline \multirow[b]{2}{*}{ Condition } & \multicolumn{4}{|l|}{ Hold } & \multicolumn{4}{|c|}{ Movement } & \multicolumn{2}{|c|}{ Orientation } \\
\hline & Partial & Omission & Incorrect & Ill-defined & Partial & Omission & Incorrect & Ill-defined & Incorrect & Ill-defined \\
\hline Perceptually similar & 83 & 17 & 0 & 0 & 38 & 38 & 24 & 0 & 43 & 57 \\
\hline Perceptually different & 64 & 9 & 27 & 0 & 17 & 44 & 33 & 6 & 53 & 47 \\
\hline Overall percentage & 71 & 10 & 19 & 0 & 25 & 47 & 25 & 3 & 50 & 50 \\
\hline
\end{tabular}

Table 4. Distribution of error types observed in BW's object use (shown as percentages)

\begin{tabular}{|c|c|c|c|c|c|c|c|c|c|c|}
\hline \multirow[b]{2}{*}{ Condition } & \multicolumn{4}{|l|}{ Hold } & \multicolumn{4}{|c|}{ Movement } & \multicolumn{2}{|c|}{ Orientation } \\
\hline & Partial & Omission & Incorrect & Ill-defined & Partial & Omission & Incorrect & Ill-defined & Incorrect & Ill-defined \\
\hline Perceptually similar & 63 & 12 & 25 & 0 & 41 & 53 & 6 & 0 & 60 & 40 \\
\hline Perceptually different & 73 & 9 & 18 & 0 & 15 & 67 & 15 & 3 & 58 & 42 \\
\hline Overall percentage & 68 & 11 & 21 & 0 & 31 & 54 & 11 & 4 & 59 & 41 \\
\hline
\end{tabular}

different but related exemplars. With the dissolution of conceptual knowledge, the ability to generalize in this way is reduced. Therefore, if the patients' degree of semantic impairment differed, so might the ability to generalize, even to perceptually similar exemplars. Once again, we conclude that this factor may have played some role but is probably not a complete explanation. It is true that, on the general measures of semantic memory shown in Table 1, JH performed slightly better than $\mathrm{BW}$, but both were extremely impaired, performing at or close to chance on the word-picture matching assessment, for example. These same two patients also participated in a laboratory-based set of experiments on use and knowledge of objects (S. Bozeat et al., 2001, unpublished data) in which they were both severely, and equally, impaired. A third possibility relates to the patients' use of other exemplars in their own homes. It was noted that $\mathrm{JH}$ possessed different examples of some objects; although we endeavoured to assess use of the particular exemplars that she used regularly, it is possible that she was also using some alternative versions as well, either in her own home or at her daughter's house next door. It seems possible, therefore, that the wider variety of exemplars encountered might encourage generalization to our experimental items.

This study confirms previous observations that patients with semantic dementia remain able to use a limited number of objects at home late into the course of the disease (Funnell, 2001). Our results suggest that it is personal familiarity rather than context that supports this ability. We note that Snowden et al.'s (1994) study on object recognition revealed effects of both variables. It is possible that the incongruous comparison context used by Snowden et al. (another room in the patient's house) may have acted as a form of distractor condition, whereas a clinical testing room may be a more neutral location. Furthermore, in the study by Snowden et al. the objects were not only placed in a different room but also with objects of a different purpose which may have led to an additional object distractor effect.

How does personal familiarity give rise to the difference between the patients' use of their own objects and alternative exemplars of the same items? There are at least two possible mechanisms. The first builds on the notion that object use is heavily reliant on semantic knowledge. Repeated experience with the same object may boost degraded conceptual representations (Snowden et al., 1994) or help to maintain a very specific form of representation whilst the remainder of the rich semantic knowledge for that concept degrades away. This exemplar-specific representation would be extremely impoverished but might provide enough 'semantic' information to enable successful use. Alternatively, the repeated use of a specific object may establish a set of automatic, stereotyped responses that are triggered by that particular object without activation of, or reliance on, semantic knowledge. The fact that neither patient was able to generalize well to perceptually different exemplars of familiar objects suggests very little conceptual knowledge remaining for these items and thus seems to fit better with the second hypothesis. We note, however, that even extremely degraded conceptual knowledge is unlikely to be zero, and that these two explanations are not in fact mutually exclusive but may both have a role to play.

Although our discussion has concentrated on the theoretical interest of this study, there are also some important clinical and practical implications. If we can understand more about the cognitive processes involved in object use and the factors that enhance these, it may be possible to optimize the patients' object use environment and, therefore, help them to maintain a degree of independence for as long as possible. 


\section{Acknowledgements}

We are grateful to BW and JH for taking part in this study, and also to their husbands for advice on object selection and for providing access to the objects.

\section{References}

Bozeat S, Lambon Ralph MA, Garrard P, Patterson K, Hodges JR. Non-verbal semantic impairment in semantic dementia. Neuropsychologia 2000; 38 1207-15.

Funnell E. From objects to properties: Evidence for spreading semantic activation in a case of semantic dementia. Memory 1995; 3: 497-519.

Funnell E. Evidence for scripts in semantic dementia: implications for theories of semantic memory. Cognitive Neuropsychology 2001; 18: 323-41.

Graham KS, Lambon Ralph M, Hodges JR. Determining the impact of autobiographical experience on "meaning": new insights from investigating sports related vocabulary and knowledge in two cases with semantic dementia. Cognitive Neuropsychology 1997; 14: 801-37.

Hamanaka T, Matsui A, Yoshida S, Nakanishi M, Fujita K, Banno T. Cerebral laterality and category-specificity in cases of semantic memory impairment with PET findings associated with identification amnesia for familiar faces. Brain and Cognition 1996; 30: 368-72.

Hodges JR, Miller BL. The classification, genetics and neuropathology of frontotemporal dementia. Introduction to the special topic papers: part 1. Neurocase 2001; 7: 31-5.

Hodges JR, Patterson K, Oxbury S, Funnell E. Semantic dementia: progressive fluent aphasia with temporal lobe atrophy. Brain 1992; 115 $1783-1806$.

Hodges JR, Garrard P, Patterson K. Semantic dementia. In: Kertesz A, Munoz DG, editors. Pick's disease and Pick complex. New York: Wiley-Liss, 1998: 83-104.

Hodges JR, Spatt J, Patterson K. What and how: Evidence for the dissociation of object knowledge and mechanical problem solving skills in the human brain. Proceedings of the National Academy of Science 1999; 96: 775-84.

Hodges JR, Bozeat S, Lambon Ralph MA, Patterson K, Spatt J. The role of conceptual knowledge in object use: evidence from semantic dementia Brain 2000; 123: 1913-25.

Howard D, Patterson K. Pyramids and Palm Trees: a test of semantic access from pictures and words. Bury St Edmunds, Suffolk: Thames Valley Test Company, 1992.

Lambon Ralph MA, Howard D. Gogi aphasia or semantic dementia? Simulating and assessing poor verbal comprehension in a case of progressive fluent aphasia. Cognitive Neuropsychology 2000; 17: 437-65.

Lauro-Grotto R, Piccini C, Shallice T. Modality-specific operations in semantic dementia. Cortex 1997; 33: 593-622.
Mummery CJ, Patterson K, Hodges JR, Wise RJS, Price CJ. Functional neuroanatomy of the semantic system: divisible by what? Journal of Cognitive Neuroscience 1998; 10: 766-77.

Mummery CJ, Patterson K, Wise RJS, Price CJ, Hodges JR. Disrupted temporal lobe connections in semantic dementia. Brain 1999; 122: 61-73.

Raven JC. Coloured progressive matrices sets A, AB, B. London: H. K. Lewis, 1962.

Schwartz MF, Buxbaum LJ. Naturalistic action. In: Rothi LJG, Heilman KM, editors. Apraxia: the neuropsychology of action. Hove: Psychology Press, 1997: 269-89.

Snowden JS, Goulding PJ, Neary D. Semantic dementia: A form of circumscribed cerebral atrophy. Behavioural Neurology 1989; 2: 167-82.

Snowden J, Griffiths H, Neary D. Semantic dementia: Autobiographical contribution to preservation of meaning. Cognitive Neuropsychology 1994; 11: $265-88$.

Snowden JS, Griffiths HL, Neary D. Semantic-episodic memory interactions in semantic dementia: implications for retrograde memory function. Cognitive Neuropsychology 1996; 13: 1101-37.

Warrington EK. Selective impairment of semantic memory. Quarterly Journal of Experimental Psychology 1975; 27: 635-57.

Warrington EK, James M. The Visual Object and Space Perception battery. Bury St Edmunds: Thames Valley Test Company, 1991.

Wechsler DA. Wechsler Adult Intelligence Scale-Revised. Test manual. New York: Psychological Corporation, 1981.

\section{Appendix}

\begin{tabular}{|c|c|}
\hline BW & $\mathrm{JH}$ \\
\hline Cafetière & $\mathrm{Comb}^{\mathrm{a}}$ \\
\hline Grater & Grater \\
\hline Jug & Hairbrush $^{\mathrm{a}}$ \\
\hline Kettle & Hairdryer $^{\mathrm{a}}$ \\
\hline Knife & Kettle \\
\hline Potato masher & Knife \\
\hline Potato peeler & Potato masher \\
\hline Scissors & Scissors \\
\hline Sieve & Serving spoon \\
\hline Spatula & Sieve \\
\hline Tea pot & Slatted spoon \\
\hline Tea strainer & Spatula \\
\hline Washing-up brush & Tin opener \\
\hline Whisk & Washing-up sponge \\
\hline Wooden spoon & Whisk \\
\hline
\end{tabular}

${ }^{a}$ Although these objects are not typical kitchen objects, they were reported to be kept and used in the kitchen by JH. 


\title{
The influence of personal familiarity and context on object use in semantic dementia
}

\section{S. Bozeat, M. A. Lambon Ralph, K. Patterson}

\section{and J. R. Hodges}

\begin{abstract}
Several laboratory experiments assessing the ability of patients with semantic dementia to use familiar objects have revealed deficits corresponding directly to the patients' remaining conceptual knowledge for the same items. The same patients, however, were reported to demonstrate normal use of some objects relevant to their everyday lives. The study reported here was designed to explore this apparent discrepancy by examining the influence of personal familiarity with object exemplars, and of the contexts in which they are typically used. Two patients with severe semantic impairment were given single objects that they were still using at home on a regular basis and asked to demonstrate the use of each. Performance on these items was compared with use of perceptually similar and perceptually different exemplars of the same objects. All three sets were tested in the patients' own homes and also in the laboratory. Both patients were significantly more successful at using their own objects than the perceptually different exemplars, while an advantage for 'own' relative to similar exemplars characterized one of the two patients. Familiar home context had no impact on performance. The results suggest that repeated experience with personally familiar objects helps to maintain appropriate responses to them in the face of severely degraded conceptual knowledge.
\end{abstract}

Journal

Neurocase 2002; 8: 127-34

\section{Neurocase Reference Number:}

$\mathrm{O} 250$

Primary diagnosis of interest

Semantic dementia

\section{Author's designation of case}

$\mathrm{JH}, \mathrm{BW}$

\section{Key theoretical issue}

- The processes involved in object use

Key words: semantic dementia; object use; semantic memory; autobiographical experience

\section{Standardized assessment}

Pyramids and Palm Trees test, Wechsler Adult Intelligence Scale-Revised

(WAIS-R), Visual Object and Space Perception battery, Raven's matrices

\section{Other assessment}

Assessment of object use

\section{Lesion location}

- Temporal lobe

\section{Lesion type}

Atrophy

Language

English 